[0212-7199 (2007) 24: 6; pp 292-299] ANALES DE MEDICINA INTERNA Copyright (C) 2007 ARAN EDICIONES, S.L.

AN. MED. INTERNA (Madrid) Vol. 24, N. ${ }^{\circ}$ 6, pp. 292-299, 2007

\title{
Infecciones por clamidias
}

\section{B. ROCA}

Servicio de Medicina Interna e Infecciones. Hospital General de Castellón. Universidad de Valencia
CHLAMYDIAL INFECTIONS

\section{RESUMEN}

Las clamidias son un grupo peculiar de bacterias, cuyo ciclo vital transcurre en parte en el interior de las células. Tienen la propiedad de adherirse a determinados epitelios, en los que pueden acabar produciendo infecciones. Existen tres especies patógenas para el hombre:

Chlamydia trachomatis, que produce infecciones fundamentalmente en los epitelios genital y urinario, y C. psittaci y $C$. pneumoniae, que producen fundamentalmente infecciones respiratorias. A las dos últimas se les clasifica en la actualidad en un género diferente, el de las Chlamydophilas.

PALABRAS CLAVE: Palabras clave: Clamidias. Infecciones urinarias. Infecciones genitales. Infecciones respiratorias.

\begin{abstract}
Chlamydiae are a singular group of bacteria, with a vital cycle that is intracellular in part. These microorganisms adhere to epithelia, where they may provoke infection. There are three pathogenic species for humans: Chlamydia trachomatis, which produce infections mainly in the genital and urinary tracts, and C. psittaci and C. pneumoniae, which mostly produce respiratory infections. The last two microorganisms are now classified in a different genus, known as Chlamydophila.
\end{abstract}

KEY WORDS: Chlamydiae. Urinary tract infection. Genital infection. Respiratory tract infection.

Roca B. Infecciones por clamidias. An Med Interna (Madrid) 2007; 24: 292-299.

\section{INTRODUCCIÓN}

Las clamidias son un grupo especial de bacterias. Poseen ADN y ARN, ribosomas similares a los de las bacterias Gram negativas y pared celular, pero tienen un ciclo vital peculiar, que transcurre en buena parte en el interior de las células.

$\mathrm{Su}$ forma contagiosa, denominada cuerpo elemental, está adaptada a la vida extracelular. Una vez alcanza a un huésped adecuado se adhiere a la superficie de determinados tejidos, generalmente epitelios columnares o transicionales, y penetra en las células de los mismos por medio de un fagosoma. Cuando lleva unas cuantas horas en el interior de las células se transforma en el denominado cuerpo reticulado, que está adaptado a la vida intracelular. En dicho lugar experimenta repetidas divisiones binarias hasta ocupar la mayor parte de la célula y adoptar el conjunto de microorganismos una morfología peculiar conocida con el nombre de inclusión, la cual es resistente a la acción de los lisosomas. Varias horas más tarde los cuerpos reticulados, todavía en el interior las inclusiones, se transforman en cuerpos elementales. Finalmente las inclusiones se rompen y liberan al exterior de la célula nuevos cuerpos elementales, que pueden contagiar a otras células y a otros huéspedes, e inician de ese modo un nuevo ciclo vital (1).

Existen tres especies de clamidias patógenas para el hombre: Chlamydia trachomatis, $C$. psittaci y $C$. pneumoniae. Una cuarta especie, $C$. pecorum sólo parece patógena para algunos animales.

Trabajo aceptado: 24 de enero de 2007 
C. trachomatis es patógena sólo para el hombre. Hace más de 50 años se identificó como el agente causante del tracoma, y posteriormente se comprobó también su papel en diversas enfermedades de transmisión sexual (ETS) y algunas infecciones perinatales. Existen al menos 20 serotipos, la mayoría de los cuales se relaciona con alguna enfermedad concreta (Tabla I).

\section{TABLA I}

SEROTIPOS DE C. TRACHOMATIS, DE ACUERDO CON LA CLASIFICACIÓN DE WANG Y GRAYSTON, Y ENFERMEDADES QUE PRODUCEN

\begin{tabular}{lc}
\hline Serotipos & Enfermedades \\
\hline$A_{1}, B_{1}$ Ba y $C$ & Tracoma \\
$D$ a K & Enfermedades de transmisión sexual (ETS) \\
$L_{1} L_{2}$ y $L_{3}$ & Linfogranuloma venéreo y proctocolitis hemorrágica \\
\hline
\end{tabular}

Las cepas que producen linfogranuloma venéreo se diferencian de las otras clamidias en que poseen una mayor capacidad invasiva, afectan a los ganglios linfáticos y crecen bien en los cultivos celulares. El resto de cepas suele afectar únicamente a los epitelios columnares superficiales de las mucosas.

C. psittaci está ampliamente distribuida en la naturaleza, y produce diversas infecciones en distintas especies de animales. Para el hombre únicamente son patógenas las especies que afectan a las aves. Produce un cuadro febril acompañado en muchos casos de una neumonía.

C. pneumoniae, conocida también con el nombre de agente "Taiwan Acute Respiratory" (TWAR), es un microorganismo difícil de cultivar, que produce infecciones respiratorias altas y neumonías, especialmente en niños y adultos jóvenes. Parece patógeno únicamente para el hombre, y se transmite por vía respiratoria, entre personas que están en estrecho contacto. Algunos estudios sugieren que este germen puede tener algún papel en la etiopatogenia de la arteriosclerosis.

\section{INFECCIONES GENITALES POR CHLAMYDIA TRACHOMATIS}

En los adultos Chlamydia trachomatis produce un espectro de manifestaciones clínicas muy similar al que ocasiona Neisseria gonorrhoeae, es decir, uretritis, proctitis y conjuntivitis en hombres y mujeres, epididimitis en hombres, y bartolinitis, cervicitis, salpingitis y perihepatitis en mujeres. Ambos microorganismos pueden ocasionar también artritis séptica. En general, los síntomas que provoca $C$. trachomatis son menos intensos que los de $N$. gonorrhoeae; de hecho, muchas infecciones por $C$. trachomatis son totalmente asintomáticas (2). La cervicitis en mujeres y la uretritis en hombres con frecuencia está producida simultáneamente por $C$. trachomatis y $N$.gonorrhoeae.

\section{EPIDEMIOLOGÍA}

C. trachomatis es la principal causante de ETS de etiología bacteriana en los países desarrollados. En las consultas especializadas pueden suponer hasta la cuarta parte de casos atendidos. La incidencia de estas infecciones varia sustancialmente de unos grupos de población a otros, pero en general son más frecuentes alrededor de los 20 años de edad. Son algo más comunes en las mujeres que en los varones (3). La utilización de anticonceptivos orales y la presencia de ectopia cervical favorecen su transmisión. Las infecciones recurrentes son habituales (4).

\section{PATOGENIA}

C. trachomatis infecta preferentemente el epitelio columnar de las mucosas de los ojos, las vías respiratorias y los genitales. Esta infección induce inmunidad, pero a menudo persiste durante meses o años, si el paciente no recibe tratamiento.

Las infecciones asintomáticas u oligosintomáticas de las trompas de Falopio provocan inflamación crónica y desestructuración de las mismas que puede acabar ocasionando infertilidad. La etiopatogenia de este proceso no es bien conocida, aunque se cree que podría estar relacionado con una reacción autoinmune desencadenada por determinadas proteínas del germen.

\section{MANIFESTACIONES CLÍNICAS}

A continuación estudiamos las diversas manifestaciones clínicas que puede producir C. trachomatis en los genitales (5).

\section{Uretritis no gonocócica y postgonocócica}

Uretritis no gonocócica (UNG) es un diagnóstico de exclusión tradicionalmente aplicado a las uretritis que ocurren en los varones y que no están producidas por $N$. gonorrhoeae. Mientras que uretritis postgonocócica (UPG) se refiere a un cuadro de uretritis, también en varones, que ocurre pocas semanas después de otro cuadro de uretritis tratado con antibióticos sin actividad contra clamidias, como por ejemplo beta-lactámicos. Como el tratamiento actualmente recomendado para la infección por $N$. gonorrhoeae incluye antibióticos activos contra $C$. trachomatis, debido a la frecuente coincidencia de ambas infecciones, la incidencia de UPG ha disminuido substancialmente.

C. trachomatis ocasiona alrededor de la tercera parte de casos de UNG en los varones heterosexuales, y un porcentaje algo menor de dicha infección en los varones homosexuales. El resto de casos de UNG está producido por diversos micoplasmas, como Ureaplasma urealyticum y Mycoplasma genitalium, protozoos como Trichomonas vaginalis, o virus como el del herpes simplex.

El síntoma fundamental de la uretritis es la presencia de exudado uretral. En muchos pacientes dicho exudado sólo es perceptible al levantarse de dormir, o al exprimir la uretra. Otros síntomas frecuentes son la disuria y el prurito en el meato uretral. Dicho meato suele estar eritematoso y suele ser doloroso al tacto.

El diagnóstico de uretritis se realiza generalmente al detectar leucocitos en el exudado uretral. Una prueba sencilla y no invasiva que permite también diagnosticar la uretritis es la detección de piuria, mediante la técnica de la esterasa leu- 
cocitaria, en una muestra de orina obtenida al principio de la micción.

El diagnóstico de UNG puede realizase cuando en el exudado no se detecta $N$. gonorrhoeae, mediante tinción de Gram o mediante cultivo. La sintomatología de la uretritis que produce $C$. trachomatis suele ser menos intensa y el exudado suele ser menos purulento que los que produce $N$. gonorrhoeae, aunque la clínica no es suficientemente fiable para distinguir ambas etiologías.

Las infecciones uretrales asintomáticas por C. trachomatis son muy frecuentes, de modo que, según algunos estudios, están presentes en hasta el $10 \%$ de varones jóvenes sexualmente activos (6).

\section{Síndrome uretral en mujeres}

C. trachomatis también puede producir en las mujeres un cuadro de uretritis, similar al de los varones, caracterizado por una sintomatología parecida a la de la cistitis, consistente en disuria, polaquiuria, tenesmo vesical y piuria, en ausencia de los gérmenes que habitualmente ocasionan cistitis.

Tal como sucede en los varones, $C$. trachomatis se encuentra en la uretra de muchas mujeres que no presentan sintomatología alguna (7).

\section{Epididimitis}

C. trachomatis es el principal agente etiológico de epididimitis en varones heterosexuales menores de 40 años sexualmente activos. $N$. gonorrhoeae ocasiona la mayoría del resto de casos en dicho grupo de personas. No son raras las infecciones mixtas por ambos microorganismos. Además, la epididimitis con frecuencia se acompaña de uretritis, sintomática o no.

En varones homosexuales la epididimitis está producida muchas veces por bacilos Gran negativos, especialmente coliformes, transmitidos por medio del coito anal. En varones mayores de 40 años también son los bacilos Gram negativos los principales causantes de epididimitis, muchas veces favorecidas por patología urológica preexistente.

La epididimitis producida por $C$. trachomatis se presenta con dolor escrotal unilateral, fiebre, y aumento de tamaño y dolor en el epidídimo. La intensidad del cuadro varía mucho de unos casos a otros, pero en ocasiones requiere el ingreso hospitalario. El diagnóstico diferencial de este proceso debe realizarse fundamentalmente con la torsión testicular, mediante estudios con radioisótopos, Doppler o exploración quirúrgica (8).

\section{Proctitis}

C. trachomatis produce proctitis en varones homosexuales que practican el coito anal receptivo o en mujeres heterosexuales que practican el coito anal. La mayoría de casos están producidos por los serotipos $\mathrm{D}$ a $\mathrm{K}$, pero en ocasiones también la producen los serotipos $\mathrm{L}_{1}$ a $\mathrm{L}_{3}$, que son lo que ocasionan el linfogranuloma venéreo.

La proctitis por $C$. trachomatis es oligosintomática o completamente asintomática, a diferencia de lo que sucede cuando está producida por $N$. gonorrhoeae, que produce cuadros mucho más intensos. Los síntomas más frecuentes son el dolor perianal, el tenesmo rectal y la emisión de secreciones mucosas, y ocasionalmente sanguinolentas, por el ano. La tinción de Gram de estas secreciones muestra la presencia de leucocitos. La anoscopia pone de manifiesto una ligera inflamación que afecta al ano y a la zona más distal del recto.

Los casos de proctitis producidos por los serotipos $\mathrm{L}_{1}$ a $\mathrm{L}_{3}$ tienden a ser más intensos, y no es raro que se manifiesten con ulceraciones de la mucosa, en cuyo caso debe realizarse el diagnóstico diferencial con la proctitis herpética y la enfermedad inflamatoria intestinal. Si no son correctamente tratados, estos procesos pueden complicarse con la aparición de abscesos perirrectales y fístulas perineales, en los que suele jugar un papel importante la sobreinfección por bacterias diferentes a las clamidias. Otra posible complicación es el desarrollo de estenosis rectales, que a veces son difíciles de distinguir de las neoplasias rectales (9).

\section{Síndrome de Reiter}

El síndrome de Reiter consta de conjuntivitis, artritis y erupciones mucocutáneas características. Acompaña a uretritis (o cervicitis en mujeres) y a gastroenteritis. Cuando se asocia a uretritis, $C$.trachomatis es el principal responsable de este síndrome, si bien en muchos casos la uretritis es subclínica.

Se sospecha que este síndrome tiene una patogenia autoinmune, favorecida por factores genéticos, ya que la mayoría de personas que lo presenta posee el antígeno de histocompatibilidad HLA-B27 (10).

\section{Cervicitis mucopurulenta}

La cervicitis por $C$. trachomatis es un proceso muy frecuente entre las mujeres sexualmente activas, aunque la mayoría de las veces es asintomático. El examen del cuello del útero con espéculo pone de manifiesto edema y facilidad para el sangrado en la zona de ectopia cervical, y presencia de exudado mucopurulento endocervial de color amarillento, en el que se detectan leucocitos con la tinción de Gram. El examen citológico muestra un predominio de neutrófilos, pero también puede observarse un infiltrado inflamatorio compuesto por linfocitos, plasmocitos e histiocitos (7).

\section{Enfermedad pélvica inflamatoria}

C. trachomatis provoca alrededor de la mitad de casos de enfermedad pélvica inflamatoria (EPI) en los países desarrollados. Esta enfermedad se produce como consecuencia de la diseminación intraluminal del microorganismo por todo el aparato genital femenino. A partir de una cervicitis se producen sucesivamente endometritis, salpingitis y peritonitis pélvica, aunque todos estos procesos suelen presentarse simultáneamente.

Entre las manifestaciones clínicas de la EPI figuran el dolor hipogástrico y metrorragias. A la exploración el útero, y especialmente los anejos son dolorosos. Cuando la EPI está producida por $N$. gonorrhoeae los síntomas suelen ser más intensos que con $C$. trachomatis.

Las infecciones crónicas de las trompas de Falopio por $C$. trachomatis, en muchos casos asintomáticas, son con toda 
probabilidad una de las principales causas de infertilidad y de embarazos ectópicos en las mujeres (11).

\section{Perihepatitis}

La perihepatitis o síndrome de Fitz-Hugh-Curtis fue descrita originalmente como una complicación de la EPI causada por $N$. gonorrhoeae, sin embargo en la actualidad este proceso está producido la mayoría de las veces por $C$. trachomatis. Ocurre en mujeres sexualmente activas, y clínicamente se presenta como un cuadro agudo o subagudo de dolor en el hipocondrio derecho y fiebre, en muchos casos con escasa o nula sintomatología en el hipogastrio. El diagnóstico diferencial debe realizarse con la colecistitis aguda, para lo cual es de gran utilidad la ecografía (12).

\section{Infecciones por C. trachomatis en mujeres gestantes y en recién nacidos}

En los países desarrollados, alrededor del 20\% de mujeres gestantes presenta cervicitis por $C$. trachomatis, generalmente asintomática pero fácilmente detectable mediante el examen con espéculo. Alrededor de la cuarta parte de los recién nacidos de madres infectadas desarrolla una conjuntivitis producida por el microorganismo, que se inicia una o dos semanas después del nacimiento y que con frecuencia ocasiona la producción de un abundante exudado purulento. Otros microorganismos, entre los que destacan N. gonorrhoeae, Haemophilus influenzae, Streptococcus pneumoniae y el virus del herpes simplex, también pueden provocar conjuntivitis en los recién nacidos. El diagnóstico diferencial se basa es estudios microbiológicos del exudado ocular. En las infecciones por $C$. trachomatis, con la tinción de Giemsa, se observan inclusiones en las células epiteliales, pero los cultivos y los procedimientos basados en la amplificación de ácidos nucleicos son más fiables para establecer el diagnóstico.

Un pequeño porcentaje de niños contagiados por $C$. trachomatis durante el parto desarrolla una neumonía por dicho germen. Esta infección parece favorecer el ulterior desarrollo de bronquitis y de asma.

Finalmente $C$. trachomatis también provoca algunos casos de otitis media en los recién nacidos (13).

\section{Conjuntivitis por C. trachomatis en adultos}

Ocasionalmente en adultos sexualmente activos se produce una conjuntivitis similar a la de los recién nacidos, causada por $C$. trachomatis. Generalmente es unilateral y se manifiesta con sensación de cuerpo extraño y exudado purulento. La mucosa afectada tiene un aspecto folicular y suele acompañarse de adenopatías preauriculares. Evoluciona lentamente a lo largo de meses, si no es tratada. Puede afectar a la córnea, donde suele causar vascularización y opacidades. Los adenovirus y los herpesvirus pueden producir conjuntivitis similares.

Esta infección tiende a persistir, y a recurrir si el paciente y sus compañeros sexuales no reciben un tratamiento adecuado (14).

\section{Linfogranuloma venéreo}

El linfogranuloma venéreo es una infección crónica producida por los serotipos $\mathrm{L}_{1}$ a $\mathrm{L}_{3}$ de $C$. trachomatis. Otros serotipos del germen también pueden producir la enfermedad (15). Se trata habitualmente de una enfermedad de transmisión sexual, pero también existen casos de contagio por contacto personal, a través de fómites o tras la exposición al germen en el laboratorio.

El contagio de esta enfermedad ocurre más frecuentemente en la segunda y la tercera década de la vida, coincidiendo con la mayor actividad sexual. Su incidencia es baja en los países desarrollados, y está disminuyendo en todo el mundo, pero sigue siendo endémica en muchos países, especialmente de Asia, África y América.

El riesgo de infección tras la exposición es probablemente bastante menor que con otras ETS, como la gonorrea o la sífilis. En los países desarrollados es más frecuente en varones, y ocurre especialmente en homosexuales y en personas que han estado en las regiones endémicas. El hombre es probablemente el principal reservorio de esta enfermedad.

Tres días a 3 semanas después del contagio se produce una infección genital primaria, consistente en una pequeña lesión vesicular, papular o ulcerada, rodeada de un halo eritematoso y no dolorosa. Se localiza en los genitales externos del varón o de la mujer. La mayor parte de las veces esta lesión pasa inadvertida, especialmente en las mujeres, y se resuelve espontáneamente, sin dejar cicatriz, en unos cuantos días. Esta infección primaria puede ocurrir, aunque con mucha menor frecuencia, en otros lugares como la uretra, el cuello del útero o el recto. En esta fase de la infección los síntomas sistémicos son poco frecuentes y poco intensos.

Desde el lugar de la infección primaria el microorganismo pasa a los linfáticos regionales, de modo que, 2 a 12 semanas después del contagio, acaba ocasionando una linfadenitis, generalmente en la región inguinal, consistente en el aumento progresivo del tamaño de los ganglios linfáticos, seguido del desarrollo de periadenitis, formación de mazacotes de adenopatías, y supuración de las mismas. La piel que recubre a las zonas afectadas suele estar inflamada y adherida a los tejidos subyacentes, y en la misma acaban apareciendo fístulas que drenan material purulento. Varios meses más tarde, aun sin tratamiento, el proceso tiende a regresar, y la inflamación finalmente desaparece, pero deja considerables cicatrices, muchas veces de aspecto nodular. El proceso puede afectar también al resto de territorios linfáticos de la pelvis, pero es unilateral en más de la mitad de los casos. Ocasionalmente quedan como secuelas complicaciones como fístulas perineales, elefantiasis de los genitales secundarias a la obstrucción linfática o estenosis uretrales.

El examen histológico de los ganglios linfáticos afectados muestra inicialmente la existencia de pequeños abscesos de morfología estrellada rodeados de histiocitos. Posteriormente dichos abscesos coalescen y forman grandes masas necróticas y purulentas. Finalmente se forman tejidos granulomatosos y cicatriciales que persisten indefinidamente.

Durante la fase de linfadenitis son comunes los síntomas generales, como fiebre, cefalea, anorexia y dolor difuso. En ocasiones también ocurren conjuntivitis, artritis, meningitis aséptica, encefalitis, hepatitis o eritema nudoso.

Se han descrito algunos casos de linfadenitis mediastínica, neumonitis y derrame pleural tras la inhalación de aerosoles con C. trachomatis en el laboratorio (16). 


\section{Otras infecciones}

Ocasionalmente se han comunicado casos aislados de otros tipos de infecciones, producidas por $C$. trachomatis, entre las que figuran endocarditis, peritonitis, infecciones respiratorias altas, pleuritis, periapendicitis, y neumonías en adultos. Presumiblemente estas infecciones se producen como consecuencia de la diseminación hematógena del microorganismo, a partir de infecciones genitales (17).

\section{DIAGNÓSTICO}

Existen cuatro modalidades de diagnóstico microbiológico para las infecciones por $C$.trachomatis: examen microscópico directo, cultivo, serología y detección de antígenos y de ácido nucleicos.

El examen microscópico directo consiste la detección de las inclusiones típicas de la infección en el raspado de los tejidos afectados, mediante la tinción de Giemsa. Se ha empleado sobre todo en la conjuntivitis, pero posee una sensibilidad y una especificidad limitadas, por lo que ya no suele emplearse.

$C$. trachomatis puede aislarse en cultivos celulares, pero estos procedimientos sólo están disponibles en algunos centros de referencia. Ello es debido a su alto coste y a sus considerables requerimientos tecnológicos. Además su sensibilidad es limitada.

La serología también posee escasa utilidad. Existen diversas modalidades de la misma, con diferentes sensibilidades y especificidades. La microinmunofluorescencia se encuentra entre las más recomendables, y se emplea especialmente en casos de neumonías en recién nacidos y de perihepatitis. Títulos altos de anticuerpos de tipo $\operatorname{IgM}$ frente a $C$. trachomatis detectados con esa técnica son altamente sugestivos de infección aguda por el microorganismo.

En los últimos años se han desarrollado diversas técnicas de detección de antígenos, como la de la inmunofluorescencia directa ligada a anticuerpos monoclonales específicos frente a antígenos de $C$. trachomatis, y la detección de ácidos nucleicos, por ejemplo mediante sondas de ARN o ADN acopladas a un proceso de amplificación basado en la reacción de la polimerasa en cadena (18). Este tipo de pruebas puede emplearse en cualquier muestra biológica, y posee una gran sensibilidad y especificidad, por lo que su empleo está generalizándose cada vez más (19).

\section{TRATAMIENTO}

La mayoría de infecciones genitales por $C$.trachomatis en adultos pueden tratarse con doxiciclina a la dosis de $100 \mathrm{mg}$ cada 12 horas durante una semana, o bien con azitromicina a la dosis de $1 \mathrm{~g}$ en una sola toma, ambas por vía oral. En las modalidades más intensas de infección, como en la EPI o en la epididimitis, puede emplearse doxiciclina a la misma dosis, pero durante dos semanas o bien eritromicina a la dosis de 500 mg cada 6 horas durante dos semanas, ambas por vía oral, aunque en estas infecciones siempre debe asociarse tratamiento activo contra $N$. gonorrhoeae, ya que las coinfecciones son frecuentes. En el linfogranuloma venéreo pueden emplearse estos mismos antibióticos, a las mismas dosis, pero durante 3 semanas. Los fracasos de estos tratamientos son excepciona- les, ya que $C$. trachomatis es sistemáticamente susceptible a dichos antibióticos.

Las fluorquinolonas, por ejemplo ofloxacino $300 \mathrm{mg}$ cada 12 horas, también parecen eficaces para tratar las infecciones por C.trachomatis.

En mujeres embarazadas el antibiótico de elección es eritromicina, aunque también se ha empleado con éxito azitromicina, e incluso amoxicilina a la dosis de $500 \mathrm{mg}$ cada 8 horas por vía oral durante 10 a 20 días, dependiendo de la modalidad concreta de infección. Las tetraciclinas, como doxiciclina, y las fluorquinolonas están contraindicadas en las mujeres gestantes (20).

En recién nacidos suele emplearse eritromicina a la dosis de $50 \mathrm{mg}$ por $\mathrm{Kg}$ de peso al día, repartida en cuatro tomas, durante 2 semanas.

Dada la contagiosidad de $C$. trachomatis y la frecuente existencia de infecciones oligosintomáticas e incluso completamente asintomáticas, es conveniente que sean evaluados y tratados contra la infección tanto los compañeros sexuales de los pacientes afectados, como los padres de los recién nacidos con infecciones por dicho germen, incluso si están asintomáti$\cos (21,22)$.

\section{PREVENCIÓN}

Por ahora no existen vacunas eficaces para prevenir las infecciones por C. trachomatis. El diagnóstico y el tratamiento precoz es la mejor forma de evitar su transmisión. La actual disponibilidad de procedimientos diagnósticos sencillos y fiables, y la existencia de tratamientos consistentes en la administración de una sola dosis de antibiótico, son de gran utilidad para llevar a cabo programas para el control de estas infecciones (23).

La manipulación de muestras que puedan contener serotipos $\mathrm{L}_{1}$ a $\mathrm{L}_{3}$ de $C$. trachomatis en el laboratorio debe contemplar las medidas adecuadas para impedir su transmisión en dicho medio.

\section{TRACOMA}

El tracoma es una modalidad de conjuntivitis crónica producida por los serotipos A, B, Ba y C de C. trachomatis, propia de ambientes con malas condiciones higiénicas. Aunque su incidencia ha disminuido en las últimas décadas, continúa siendo una de las principales causas de ceguera en muchos países subdesarrollados de Asia y de África. Se transmite desde los ojos de las personas afectadas a los ojos de otras personas a través de las manos, de moscas, de toallas o de otros fómites. En las zonas endémicas se produce la infección por primera vez en la niñez, pero son habituales las reinfecciones. También son comunes las infecciones crónicas. Los tratamientos con corticoides pueden causar la reagudización de infecciones antiguas.

\section{CLÍNICA}

El tracoma se inicia de modo insidioso, como una conjuntivitis con pequeños folículos linfoides. La infección tiende a persistir indefinidamente, pero además en las regiones endé- 
micas continuamente se producen reinfecciones. De ese modo, más o menos tarde, acaba afectándose la cornea, en la que aparece un infiltrado inflamatorio con leucocitos y una vascularización superficial que se conoce con el nombre pannus. La inflamación continuada de la conjuntiva a lo largo de los años produce cicatrizaciones que deforman los párpados y provocan entropión (desviación del borde de los párpados hacia dentro) y triquiasis (crecimiento de las pestañas hacia el globo ocular), que dañan el globo ocular y provocan ulceraciones corneales recidivantes. Además, la destrucción de las células caliciformes de la conjuntiva, de las glándulas lagrimales y de los conductos lagrimales ocasiona una intensa sequedad ocular que provoca la opacificación y la ulceración corneal, y favorece el desarrollo de infecciones bacterianas secundarias, especialmente por Haemophilus influenzae. Con el transcurso de los años, el resultado final de todos estos procesos es la ceguera (24).

\section{DIAGNÓSTICO}

En las regiones endémicas de los países subdesarrollados el tracoma suele diagnosticarse clínicamente, cuando se dan dos de las cuatro circunstancias siguientes: presencia de folículos linfoides en la conjuntiva tarsal superior, cicatrices conjuntivales típicas, pannus, o folículos límbicos o cicatrices de los mismos (hoyuelos de Herbert).

Siempre que sea posible debe confirmarse el diagnóstico con los procedimientos descritos en el apartado de infecciones genitales por $C$. trachomatis, especialmente los basados en la detección de antígenos o de ácidos nucleicos del microorganismo.

\section{PREVENCIÓN Y TRATAMIENTO}

Por ahora no existe ninguna vacuna eficaz contra el tracoma, pero las simples medidas higiénicas generales son muy eficaces para reducir la incidencia de la enfermedad.

El tratamiento es similar al de las infecciones genitales por C. trachomatis. En distintas regiones endémicas se han llevado a cabo programas, consistentes en la aplicación de diversas modalidades de tratamiento, como pomadas con tetraciclinas o eritromicina durante varias semanas o de modo intermitente (25). La dosis única de azitromicina administrada a gran escala parece una buena opción para el control de la infección en tales regiones (26).

\section{INFECCIONES POR CHLAMYDIA PSITTACI}

La psitacosis es una enfermedad infecciosa producida por Chlamydia psittaci, que afecta principalmente a diversas especies de aves. En el hombre se manifiesta como un cuadro febril acompañado de una neumonía.

\section{EPIDEMIOLOGÍA}

La mayoría de especies de aves sufren infecciones por $C$. psittaci, aunque son los loros y los periquitos los más frecuentemente afectados. Cuando esta infección ocurre en aves dife- rentes de esas dos, se le conoce también con el nombre de ornitosis, si bien el nombre genérico de psitacosis, aplicable a todos los casos, es el más empleado. Las aves infectadas pueden morir por la enfermedad, pero normalmente sólo presentan síntomas menores o incluso están asintomáticas. Meses después de pasar la enfermedad pueden todavía albergar el germen. Diversas especies de mamíferos pueden presentar también esta infección.

El hombre se contagia habitualmente a partir de las aves, y por tanto la enfermedad ocurre en personas que de una u otra forma están en contacto con las mismas. C. psittaci se encuentra en las secreciones respiratorias, en las excretas, en las plumas y en diversos tejidos de las aves. En el hombre penetra generalmente a través de las vías respiratorias, pero también puede hacerlo a través de picaduras. También se han descrito algunos casos de transmisión nosocomial (27).

Como en el hombre en muchas ocasiones la psitacosis es oligosintomática o completamente asintomática, no se conoce con precisión su incidencia, aunque la misma parece que ha ido aumentando progresivamente a lo largo de las últimas décadas.

\section{PATOGENIA}

En el hombre, a partir de las vías respiratorias, $C$. psittaci llega a la sangre y finalmente se localiza en los alvéolos pulmonares y en las células del sistema reticuloendotelial del bazo y el hígado. La presencia del microorganismo provoca una reacción inflamatoria linfocitaria, que en los pulmones hace que los alvéolos se llenen de productos inflamatorios. En ocasiones en los tejidos afectados se observan macrófagos con inclusiones citoplásmicas característicos (inclusiones de Levinthal-Coles-Lillie).

\section{CLÍNICA}

En el hombre, las manifestaciones clínicas de la psitacosis son muy variables. Tras un periodo de incubación de una a tres semanas se inicia la enfermedad de un modo más o menos brusco con fiebre alta, escalofríos, malestar y mialgias. El pulso suele ser más lento de lo que cabría esperar con la fiebre. La cefalea está casi siempre presente, y en muchos pacientes es el síntoma principal. También pueden aparecer síntomas de encefalopatía, como desorientación, obnubilación o agitación; en los casos más severos puede llegar a ocurrir el coma. Muchos enfermos notan espasmos musculares, especialmente en la espalda.

Los síntomas respiratorios altos son comunes, pero generalmente poco intensos. No es raro que exista epistaxis y fotofobia. También es frecuente la tos, que suele ser irritativa, aunque en algunos casos es productiva de escasas cantidades de esputo purulento; el algunos casos el esputo está teñido de pequeñas cantidades de sangre. La disnea es poco común, excepto en pacientes con patología cardiaca o pulmonar preexistente.

Los síntomas digestivos, como náuseas, diarrea o dolor abdominal son habituales. Otras manifestaciones menos frecuentes son el derrame pleural, miocarditis, pericarditis, endocarditis, ictericia, erupción cutánea (máculas de Horder), tromboflebitis y embolia pulmonar. 
A la exploración suele observarse una ligera faringitis, y son muy frecuentes las adenopatías cervicales. La auscultación pulmonar suele ser muy poco llamativa; pueden existir sibilantes y sobre todo crepitantes diseminados, pero los signos de consolidación casi siempre están ausentes. Alrededor de la mitad de pacientes tiene hepatomegalia y esplenomegalia.

La psitacosis, sin tratamiento, suele durar desde unas pocas semanas hasta varios meses. En algunos pacientes se producen recrudescencias de la enfermedad durante la fase de convalecencia. Sin embargo, con toda probabilidad, esta infección deja inmunidad permanente para las reinfecciones por el mismo germen. Los casos de psitacosis contraídos a partir de los loros y los periquitos son generalmente más graves que los contraídos a partir de otras aves.

\section{DIAGNÓSTICO}

En los pacientes con psitacosis las radiografías de tórax muestran una amplia variedad de infiltrados pulmonares, generalmente intersticiales y periféricos, aunque también pueden ser nodulares y homogéneos, difusos, miliares, etc.

Los análisis suelen mostrar únicamente alteraciones inespecíficas, como una ligera leucocitosis o leucopenia, aumento de la velocidad de sedimentación globular, mínima elevación de las enzimas hepáticas o proteinuria. El líquido cefalorraquídeo puede mostrar una ligera pleocitosis mononuclear, pero por lo demás es normal.

El diagnóstico de psitacosis puede confirmarse mediante el cultivo de C. psittaci o mediante la serología. El cultivo de este microorganismo es técnicamente difícil y además peligroso para el personal que manipula las muestras, por lo que esa modalidad diagnóstica se encuentra disponible en pocos centros. La serología, especialmente la fijación de complemento, es el procedimiento diagnóstico más habitualmente empleado. Deben compararse dos muestras, una de la fase inicial de la infección y otra de la fase de convalecencia y comprobar un aumento de al menos cuatro diluciones del título de anticuerpos entre ambas para poder realizar el diagnóstico. Las infecciones por $C$. trachomatis y $C$. pneumoniae pueden dar falsos positivos. Cuando estas infecciones son posibles es conveniente utilizar la modalidad de serología de microinmunofluorescencia, con la que no se producen reacciones cruzadas con las otras clamidias. Al interpretar los resultados de la serología, debe tenerse en cuenta que el tratamiento antibiótico puede atrasar substancialmente la aparición de anticuerpos.

Con toda probabilidad, en un futuro cercano, las técnicas moleculares de detección de antígenos o ácidos nucleicos facilitarán el diagnóstico de esta infección.

$\mathrm{El}$ antecedente de haber estado en contacto con aves es de gran utilidad en muchos casos para considerar el diagnóstico de psitacosis. De todos modos, el diagnóstico diferencial de esta infección es muy amplio, e incluye diversas viriasis, el resto de neumonías atípicas y, en muchas ocasiones, las meningitis.

\section{TRATAMIENTO}

Las tetraciclinas son sistemáticamente eficaces en la psitacosis. Puede emplearse por ejemplo doxiciclina a la dosis de $100 \mathrm{mg}$ cada 12 horas. Habitualmente la respuesta es excelen- te, con la práctica desaparición de todos los síntomas en unos pocos días. No obstante se recomienda mantener el tratamiento durante dos o tres semanas. Cuando no pueden utilizarse las tetraciclinas se puede emplear eritromicina (28).

\section{INFECCIONES POR CHLAMYDIA PNEUMONIAE}

Chlamydia pneumoniae es otro agente causal de infecciones respiratorias en adultos. Se distingue de las otras clamidias por la morfología de sus cuerpos elementales y por su ADN. Es más difícil de cultivar que el resto de clamidias.

\section{EPIDEMIOLOGÍA}

Las infecciones por $C$. pneumoniae comienzan a ocurrir a partir de los últimos años de la niñez, su mayor incidencia tiene lugar en varones jóvenes, pero puede presentarse en adultos de cualquier edad (29). C. pneumoniae es un germen ubicuo, ya que más del $40 \%$ de la población adulta posee anticuerpos contra el mismo. Con toda probabilidad se transmite de persona a persona. Puede ocasionar epidemias de infecciones respiratorias altas y de neumonías, especialmente en grupos de personas que viven es estrecho contacto, como en los acuartelamientos, o en el ámbito familiar. Las reinfecciones son comunes.

\section{PATOGENIA}

La patogenia de la infección por C. pneumoniae se conoce peor que la de las otras clamidias. La infección se inicia en las vías aéreas altas y en muchas personas permanece allí el microorganismo indefinidamente. En algunos pacientes $C$. pneumoniae se desplaza, probablemente a través de la sangre, a otros lugares del organismo. Una proteína de la membrana de C. pneumoniae es capaz de inducir una respuesta autoinmune, que puede atacar a diversos tejidos del organismo.

\section{CLÍNICA}

C. pneumoniae puede producir otitis, sinusitis, faringitis, bronquitis y neumonías. Con frecuencia se asocian varias de esas afecciones o incluso todas ellas en un mismo pacientes. Son comunes la fiebre, la tos irritativa y el malestar general. La neumonía es generalmente atípica, similar a la que produce, por ejemplo, Mycoplasma pneumoniae. No suele haber leucocitosis, la auscultación pulmonar suele ser prácticamente normal, y en las radiografías suelen aparecer infiltrados intersticiales.

Se han descrito casos aislados de encefalitis, síndrome de Guilain-Barré, artritis, eritema nudoso, endocarditis, miocarditis y pericarditis producidos por C. pneumoniae.

La primera infección por $C$. pneumoniae suele provocar una clínica más intensa que las reinfecciones. En ancianos las neumonías por este germen pueden ser especialmente graves.

Diversos estudios epidemiológicos han mostrado una asociación entre la serología positiva para $C$. pneumoniae y la arteriosclerosis. Incluso con diversas técnicas, como las basadas en la hibridación de ADN, la inmunohistoquimia o los 
cultivos, se ha detectado la presencia del microorganismo en la placa de ateroma (30). Finalmente algunos estudios sugieren un efecto beneficioso de los antibióticos activos contra $C$. pneumoniae en la evolución de la arteriosclerosis, aunque por ahora no existen conclusiones definitivas al respecto (31).

También se ha atribuido a $C$. pneumoniae un posible papel en la etiolopatogenia del asma, la sarcoidosis y el cáncer de pulmón (32).

\section{DIAGNÓSTICO}

Por ahora no existe ningún procedimiento sencillo y eficaz para diagnosticar la infección por C. pneumoniae. La serología está ampliamente disponible, especialmente la basada en la fijación de complemento, pero tiene el inconveniente de que no es capaz de distinguir entre $C$. pneumoniae y $C$. tra- chomatis o $C$. psittaci. Las técnicas basadas en la microinmunofluorescencia parecen proporcionar resultados algo más fiables (33).

Por otra parte, el aislamiento del germen es técnicamente complicado, y requiere cultivos celulares, por lo que no está disponible en la mayoría de los centros. Y tampoco se han desarrollado por ahora técnicas basadas en la detección de antígenos o de ácidos nucleicos del microorganismo.

\section{TRATAMIENTO}

Los macrólidos y las tetraciclinas poseen buena actividad contra $C$. pneumoniae. Puede emplearse eritromicina a la dosis de $500 \mathrm{mg}$ cada 6 horas o bien doxiciclina a la dosis de $100 \mathrm{mg}$ cada 12 horas, ambas por vía oral y durante 10 a 15 días. Las fluorquinolonas también parecen eficaces (34).

\section{Bibliografía}

1. Hammerschlag MR. The intracellular life of chlamydiae. Semin Pediatr Infect Dis 2002; 13: 239-48.

2. Butler C. Are all genital Chlamydia trachomatis infections pathogenic? Sex Transm Infect 2005; 81: 187.

3. Miller WC, Ford CA, Morris M, Handcock MS, Schmitz JL, Hobbs MM, et al. Prevalence of chlamydial and gonococcal infections among young adults in the United States. JAMA 2004; 291: 2229-36.

4. Stamm WE. Chlamydia trachomatis -the persistent pathogen: Thomas Parran Award Lecture. Sex Transm Dis 2001; 28: 684-689.

5. Young F. Sexually transmitted infections. Genital chlamydia: practical management in primary care. J Fam Health Care 2005; 15: 19-21.

6. Simpson T, Oh MK. Urethritis and cervicitis in adolescents. Adolesc Med Clin 2004; 15: 253-71.

7. Falk L, Fredlund H, Jensen JS. Signs and symptoms of urethritis and cervicitis among women with or without Mycoplasma genitalium or Chlamydia trachomatis infection. Sex Transm Infect 2005; 81: 73-8.

8. Drury NE, Dyer JP, Breitenfeldt N, Adamson AS, Harrison GS. Management of acute epididymitis: Are European guidelines being followed? Eur Urol 2004; 46: 522-4.

9. Weir E. Lymphogranuloma venereum in the differential diagnosis of proctitis. CMAJ 2005; 172: 185.

10. Reactive arthritis. A clinical approach. Orthop Nurs 2004; 23: 274-280.

11. Wiesenfeld HC, Sweet RL, Ness RB, Krohn MA, Amortegui AJ, Hillier SL. Comparison of acute and subclinical pelvic inflammatory disease. Sex Transm Dis 2005; 32: 400-5.

12. Takeshita T, Shima H, Oishi S, Machida N, Kashima R, Ohno S, Uchiyama K. Fitz-Hugh-Curtis syndrome: Hepatic capsular enhancement and diffuse gallbladder wall thickening on contrast-enhanced CT. Intern Med 2004; 43: 632-3.

13. Zar HJ. Neonatal chlamydial infections: Prevention and treatment. Paediatr Drugs 2005; 7: 103-10.

14. Clarke J. Predicting bacterial cause in infectious conjunctivitis: Chlamydia needs to be taken into account. BMJ 2004; 329: 625.

15. Spaargaren J, Fennema HS, Morre SA, de Vries HJ, Coutinho RA. New lymphogranuloma venereum Chlamydia trachomatis variant, Amsterdam. Emerg Infect Dis 2005; 11: 1090-2.

16. Mabey D, Peeling RW. Lymphogranuloma venereum. Sex Transm Infect 2002; 78: 90-2.

17. Adams EJ, Charlett A, Edmunds WJ, Hughes G. Chlamydia trachomatis in the United Kingdom: A systematic review and analysis of prevalence studies. Sex Transm Infect 2004; 80: 354-62.

18. Tan HH, Chan R. Use of polymerase chain reaction on pooled cervical swabs to detect Chlamydia trachomatis infections in female sex workers in Singapore. Singapore Med J 2005; 46: 215-8.
19. Cook RL, Hutchison SL, Ostergaard L, Braithwaite RS, Ness RB. Systematic review: Noninvasive testing for Chlamydia trachomatis and Neisseria gonorrhoeae. Ann Intern Med 2005 7; 142: 914-25.

20. Adimora AA. Treatment of uncomplicated genital Chlamydia trachomatis infections in adults. Clin Infect Dis 2002; 35 (Supl. 2): S183-S6.

21. Golden MR, Whittington WL, Handsfield HH, Hughes JP, Stamm WE, Hogben $\mathrm{M}$ et al. Expedited treatment of sex partners in cases of persistent gonorrhea and chlamydial infection. N Engl J Med 2005; 352: 676-85.

22. Erbelding EJ, Zenilman JM. Better control of sexually transmitted diseases. N Engl J Med 2005; 352: 720-1.

23. van Bergen JE, Postma MJ, Peerbooms PG, Spangenberg AC, Tjen-ATak J, Bindels PJ. Effectiveness and cost-effectiveness of a pharmacybased screening programme for Chlamydia trachomatis in a high-risk health centre population in Amsterdam using mailed home-collected urine samples. Int J STD AIDS 2004; 15: 797-802.

24. Mariotti SP. New steps toward eliminating blinding trachoma (editorial). N Engl J Med 2004; 351: 2004-7.

25. Mabey D, Solomon A. The effect of antibiotic treatment on active trachoma and ocular Chlamydia trachomatis infection. Expert Rev Anti Infect Ther 2003; 1: 209-16.

26. Solomon AW, Holland MJ, Alexander ND, Massae PA, Aguirre A, Natividad-Sancho A et al. Mass treatment with single-dose azithromycin for trachoma. N Engl J Med 2004; 351: 1962-71.

27. Hughes C, Maharg P, Rosario P, Herrell M, Bratt D, Salgado J, Howard D. Possible nosocomial transmission of psittacosis. Infect Control Hosp Epidemiol 1997; 18: 165-8.

28. Andersen AA, Vanrompay D. Avian chlamydiosis. Rev Sci Tech 2000 19: 396-404.

29. Dal Molin G, Longo B, Not T, Poli A, Campello C. A population based seroepidemiological survey of Chlamydia pneumoniae infections in schoolchildren. J Clin Pathol 2005; 58: 617-20.

30. Liu C, Waters DD. Chlamydia pneumoniae and atherosclerosis: from Koch postulates to clinical trials. Prog Cardiovasc Dis 2005; 47: 2309.

31. Grayston JT, Kronmal RA, Jackson LA, Parisi AF, Muhlestein JB, Cohen JD et al. Azithromycin for the secondary prevention of coronary events. N Engl J Med 2005; 352: 1637-45.

32. Laurila AL, Von Hertzen L, Saikku P. Chlamydia pneumoniae and chronic lung diseases. Scand J Infect Dis Suppl 1997; 104: 34-6.

33. Fernández F, Gutiérrez J, Mendoza J, Linares J, Soto MJ. A new microimmunofluorescence test for the detection of Chlamydia pneumoniae specific antibodies. J Basic Microbiol 2004; 44: 275-9.

34. Hammerschlag MR. Advances in the management of Chlamydia pneumoniae infections. Expert Rev Anti Infect Ther 2003; 1: 493-503. 